\section{Christoph Bachmann}

\section{Einleitung}

Etwa die Hälfte aller Patienten mit chronischer Herzinsuffizienz sind ältere Frauen mit einer langen Vorgeschichte von arterieller Hypertonie. Sie leiden an diastolischer Dysfunktion mit bestehender HFpEF. Die gegenwärtig gültigen Guidelines zur Behandlung dieser Patienten empfehlen eine optimale Behandlung der zugrunde liegenden Erkrankung, d.h. auch der Risikofaktoren, und eine Anpassung der Lebensgewohnheiten, wie z.B. regelmässiges moderates Training.

Studien $[1,2]$ haben gezeigt, dass der Weissdorn-Extrakt WS 1442 (siehe Kasten) die Symptome und die Trainingstoleranz bei Patienten mit Herzinsuffizienz (NYHA II-III) verbessern. Bisher wurden jedoch keine Studien mit HFpEF-Patienten durchgeführt.

WS 1442 hat folgende Eigenschaften:

- Erhöhung der Dicke und Verminderung der Starre der Glykokalyx [3];

- Aktivierung der NO-Synthase im Endothelium und in den Erythrozyten [4-6];

- Erhöhung der endothelialen NOFreisetzung und Verbesserung der Endothelium-abhängigen Arterienfunktion bei Alterung und Hypertonie (Tiermodell) $[7,8]$;

Zusammenfassung der Studie von Härtl S, et al: Effects of endurance exercise training and Crataegus extract WS ${ }^{\circledR}$ 1442 in patients with heart failure with preserved ejection fraction - a randomized controlled trial. Sports 2014;2:5975. Übersetzung und Kürzungen: Dr. C. Bachmann.

\section{Aus der Forschung}

Schweiz Z Ganzheitsmed 2015;27:264-267

Online publiziert: 7. September 2015

\title{
Wirkung von Ausdauertraining und Weissdorn-Extrakt WS 1442 bei Patienten mit Herzfehler mit bestehender Auswurffraktion - eine randomisierte kontrollierte Studie
}

Gegenwärtige Guidelines für Patienten mit diastolischer Dysfunktion und bestehender linksventrikulärer Auswurffraktion (Heart Failure with preserved left ventricular Ejection Fraction (HFpEF)) empfehlen neben einer optimalen Behandlung der Symptome das Ausschalten von Risikofaktoren sowie ein moderates körperliches Training. In der vorliegenden Studie wurde zum ersten Mal untersucht, ob neben diesen Massnahmen die zusätzliche Gabe des Weissdorn-Extrakts WS 1442 einen weiteren Nutzen bringt.

- Erhöhung der Kontraktionskraft von Herzmuskeln durch einen ATPunabhängigen Mechanismus [9];

- positive Wirkung auf die Nachlast (Tiermodell) [10-12];

- Erhöhung der Mitochondrien-Dichte in den Skelettmuskeln und des Faserdurchmessers [13].

Die vaskulären Wirkungen hängen stark vom hohen Gehalt an oligomeren Procyanidinen im WS $1440 \mathrm{ab}$ [5].

Das Ziel der vorliegenden Studie war es, die Wirkung von zusätzlich zu einer kardiologischen Standardbehandlung verabreichtem WS 1442 und gleichzeitigem körperlichen Training auf Symptome und Lebensqualität, Trainingsleistung sowie kardiale, vaskuläre und muskuläre Funktion bei Patienten mit HFpEF, Stadium NYHA II, zu erfassen.

\section{Studie}

Für diese monozentrische, prospektive, randomisierte offene PhaseIV-Studie wurden je 70 Männer und Frauen mit diagnostizierten Herzbe- schwerden des Stadiums NYHA II, einer echographisch festgestellten linksventrikulären Auswurffraktion (LVEF) von $>40 \%$, NT-proBNP (N-terminales pro-brain natriuretic peptide) $<450$ $\mathrm{ng} / \mathrm{ml}$ und weniger als $2 \mathrm{~h}$ physischer Aktivität pro Woche rekrutiert.

Ausschlusskriterien waren: Schwere oder akute kardiale oder allgemeine Erkrankungen, Vorhofflimmern, Herzschrittmacher oder implantierbarer Kardioverter-Defibrillator, eine signifikante Verschlechterung bestehender kardialer Probleme innerhalb der letzten Woche vor der Studie sowie Behandlung mit Herzglykosiden, Weissdorn oder anderen herzaktiven Arzneipflanzen.

Die rekrutierten Patienten wurden in die Gruppen i) kardiale Behandlung, körperliches Training und WS $1442(\mathrm{n}=70)$ und ii) kardiale Behandlung, körperliches Training ohne WS $1442(\mathrm{n}=70)$ randomisiert. Die demographischen Daten der Probanden unterschieden sich nicht wesentlich. Die Probanden vollführten 8 Wochen lang zweimal wöchentlich während $90 \mathrm{~min}$ ein genau definiertes, moderates Lauf-

\section{KARGER}

(C) 2015 S. Karger GmbH, Freiburg

Fax +497614520714 
bandtraining. Weiter erhielt Gruppe 1 neben ihrer kardiologischen Grundbehandlung während 8 Wochen zweimal täglich 450 mg WS 1442.

\section{Zielvariablen}

Als Zielvariablen dienten:

- Lebensqualität: Kansas City CardiomyopathyQuestionnaire (KCCQ);

- Trainingstoleranz: Freiburger Fragebogen zur körperlichen Aktivität, 2-km-Gehtest, Herz-Lungen-Trainingsprotokoll mit einem Laufband.

\section{Resultate}

Die Resultate sind in Tabelle 1 ersichtlich. Die Patienten in der Gruppe mit zusätzlicher Gabe von WS 1442 hielten sich etwas weniger gut an die Trainingsvorgaben als die Patienten in der Gruppe ohne WS 1442. 79\% der Patienten mit zusätzlicher Behandlung nahmen an mehr als 10 Trainingseinheiten teil, in der anderen Gruppe waren es 89\% der Patienten.

\section{Behandlungsgruppe ausschliesslich Training}

Die Symptome und die körperliche Ausdauer verbesserten sich bis zur achten Woche der Behandlung/des Trainings. Die Punktezahl auf dem KCCQ verbesserte sich signifikant, ebenso die Trainingszeit, der Laktatgrenzwert, die Leistungsfähigkeit der Muskeln, die subjektive Anstrengung beim Trainingsniveau 3, die körperliche Aktivität und der Zeitaufwand für den 2-km-Gehtest. Die Trainingszeit auf dem Laufband, die Herzleistung und die Muskelleistung bei maximalem Training nahmen zu. Dabei blieb die relative Sauerstoffaufnahme unverändert bei $22,2 \mathrm{ml} / \mathrm{min} / \mathrm{kg}$. Die subjektive Anstrengung auf dem Trainingsniveau 5 und bei maximalem Training nahm nicht signifikant $\mathrm{zu}$.

Die Abnahme der Sauerstoffsättigung der Skelettmuskel durch das

Tab. 1. Resultate (Mittelwerte \pm Standardabweichung) der achtwöchigen Behandlung bezüglich der Symptome und der Trainingskapazität

\begin{tabular}{|c|c|c|c|c|}
\hline & \multicolumn{4}{|c|}{ Behandlung } \\
\hline & \multicolumn{2}{|c|}{ Training alleine } & \multicolumn{2}{|c|}{ Training + WS 1442} \\
\hline & Baseline & Veränderung & Baseline & Veränderung \\
\hline \multicolumn{5}{|l|}{ KCCQ } \\
\hline Körperliche Einschränkung & $85 \pm 17$ & $4 \pm 15^{\mathrm{a}}$ & $82 \pm 17$ & $6 \pm 15$ \\
\hline Symptomstabilität & $57 \pm 29$ & $9 \pm 35$ & $60 \pm 29$ & $3 \pm 29$ \\
\hline Symptome & $76 \pm 18$ & $5 \pm 14^{\mathrm{a}}$ & $70 \pm 22$ & $10 \pm 17$ \\
\hline Selbstvertrauen & $70 \pm 21$ & $2 \pm 17$ & $70 \pm 20$ & $3 \pm 15$ \\
\hline Soziale Einschränkung & $79 \pm 23$ & $5 \pm 16^{\mathrm{a}}$ & $76 \pm 25$ & $9 \pm 20$ \\
\hline Lebensqualität & $71 \pm 24$ & $9 \pm 17^{a}$ & $69 \pm 23$ & $11 \pm 18$ \\
\hline \multicolumn{5}{|l|}{ Trainingskapazität } \\
\hline $\begin{array}{c}\text { Freiburger Fragebogen über } \\
\text { körperliche Aktivität }\end{array}$ & $27 \pm 23$ & $14 \pm 27^{\mathrm{a}}$ & $27 \pm 26$ & $14 \pm 29$ \\
\hline 2-km-Gehtest (min) & $21,4 \pm 2,4$ & $-1,9 \pm 2,0^{\mathrm{a}}$ & $23 \pm 3,1$ & $-2,9 \pm 2,4^{\mathrm{b}}$ \\
\hline Laufbandtest (Zeit bis Ende) & $11,1 \pm 3,0$ & $1,3 \pm 2,0^{\mathrm{a}}$ & $9,6 \pm 3,7$ & $1,3 \pm 2,7$ \\
\hline
\end{tabular}

${ }^{a} \mathrm{p}<0,05$ : Signifikante Verbesserung gegenüber Baseline, kein signifikanter Mehrnutzen durch WS 1442. ${ }^{b} \mathrm{p}<0,019$ : Signifikanter Mehrnutzen mit WS 1442 gegenüber Vergleichsgruppe.

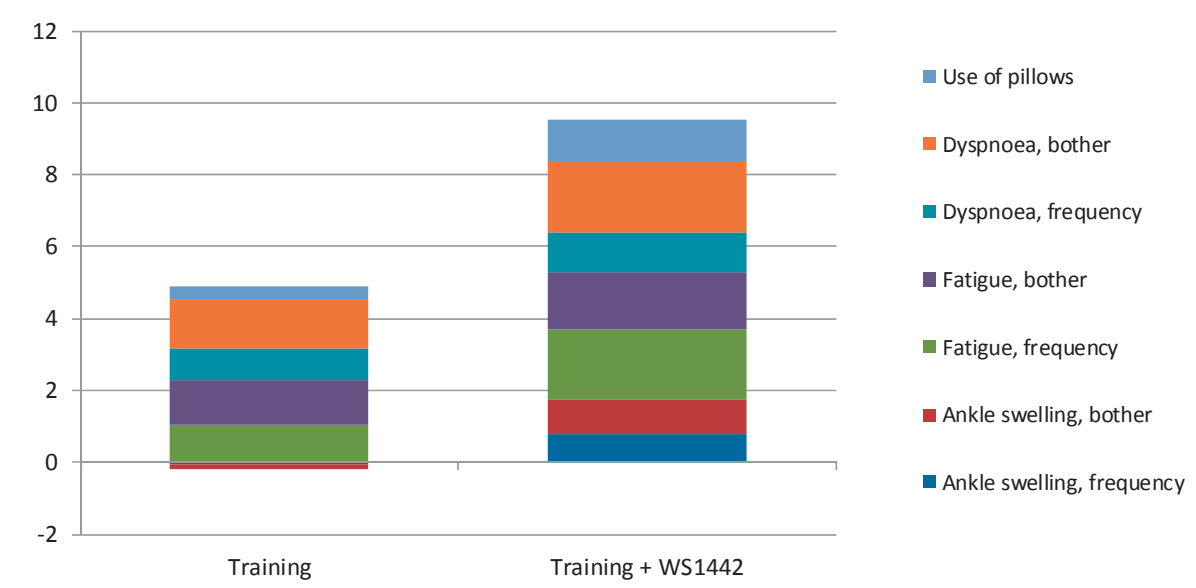

Abb. 1. Absolute Veränderung der Werte des KCCQ-Score «Symptome» während des achtwöchigen Trainings.

Training erwies sich als minimal, und das NT-proBNP nahm minimal zu.

\section{Behandlungsgruppe Training und} WS 1442

Die absolute Verbesserung der Symptome und der Lebensqualität waren tendenziell höher in der Gruppe Training und Behandlung mit WS 1442 (Tab. 1). Die Differenzierung der Werte für «Symptome» sind in Abbildung 1 ersichtlich.
Die durchschnittliche Gehzeit für die $2 \mathrm{~km}$ wurde durch die zusätzliche Gabe von WS 1442 signifikant vermindert - in der Gruppe Training ohne WS 1442 von 21 auf $20 \mathrm{~min}$ $(-8,4 \%)$ sowie in der Gruppe Training und WS 1442 von 23 auf $20 \mathrm{~min}$ $(-12,7 \% ; p=0,019)$.

Die Werte für das Laufbandtraining verbesserten sich in beiden Studiengruppen auf vergleichbare Weise.

Die subjektive Belastung, gemessen auf hohem Trainingsniveau (Niveau 
5), zeigte für die Gruppe mit WS 1442 eine tendenzielle Verbesserung.

Die Abnahme der Sauerstoffsättigung der Skelettmuskeln erwies sich vom Trainingsniveau 1 auf höhere Stufen gemessen in der Gruppe mit WS 1442 als tendenziell ausgeprägter als in der Gruppe ohne WS 1442.

\section{Verträglichkeit und Sicherheit}

Das 8 Wochen dauernde Training wurde professionell überwacht und erwies sich im Allgemeinen als sicher. Die meisten dokumentierten unerwünschten Ereignisse betrafen das muskuloskelettale System und Verletzungen, die aber alle nur unbedeutend waren. Die Frequenz solcher unerwünschter Ereignisse war in der Gruppe mit WS 1442 nicht höher (13\% der Probanden) als in der Gruppe ohne WS 1442 (17\% der Probanden). Als einziges ernsthaftes unerwünschtes Ereignis wurde eine bakterielle Urogenitalinfektion festgestellt. Die Prüfärzte sahen aber keinen Zusammenhang zwischen der Infektion und dem Prüfmedikament WS 1442. Während der Studie wurden keine Ereignisse festgehalten, die möglicherweise oder wahrscheinlich im Zusammenhang mit WS 1442 standen.

\section{Diskussion}

Die hier beschriebenen Patienten mit Herzbeschwerden und bestehender Auswurffraktion, die gemäss der aktuellen Richtlinien behandelt wurden, erlebten mit dem Ausdauertraining eine signifikante Verbesserung ihrer Symptome und der Lebensqualität. Sie erreichten dadurch Werte zwischen 80 und 90 von 100 der wichtigen Subskalen des KCCQ. Dies bedeutet, dass nur noch minimale Sym-
Kasten. Weissdorn-Extrakt WS 1442

Beim Weissdorn-Extrakt WS 1442 handelt es sich um einen Trockenextrakt aus getrockneten Blättern und Blüten verschiedener Crataegus-Arten (DEV 4-6,6:1, Lösungsmittel Alkohol 45\%/v/v). Der Extrakt ist auf 17,3-20,1\% oligomere Procyanidine standardisiert.

ptome vorhanden waren. Diese Resultate stimmen mit früheren Studien überein [14-16].

Da die Probanden dieser Studie schon durch das Ausdauertraining Werte von 80-90 bei wichtigen Subskalen des KCCQ erreichten, blieb für einen weiteren, von WS 1442 herbeigeführten Nutzen nur noch wenig Raum. In dieser Hinsicht war die Studie «underpowered», um einen statistisch signifikanten Zusatznutzen ermitteln zu können. Dies gelang jedoch trotzdem beim 2-km-Gehtest. Da der Freiburger Fragebogen die Erhöhung der körperlichen Aktivität nur in Bezug auf die Teilnahme der Probanden am Training festhielt, konnte deshalb aufgrund dieser Ergebnisse kein Gruppenunterschied festgestellt werden. Die Trainingsprotokolle wurden nur bis zu den vorher festgelegten Herzfrequenzen erstellt - und nicht bis zur Erschöpfung der Probanden. Deshalb waren die Verbesserungen auf dem Laufband moderat und zeigten keine Gruppenunterschiede. In der Gruppe mit WS 1442 wurde ein Trend zu weniger Anstrengung festgestellt, was bedeuten könnte, dass ein Training bis zur Erschöpfung einen Gruppenunterschied anzeigen könnte.

Es konnte festgestellt werden, dass Training und die zusätzliche Gabe von WS 1442 mit zunehmendem Training eine deutliche Abnahme der durchschnittlichen Sauerstoffsättigung der Skelettmuskeln bewirkte, was in der Gruppe mit Training alleine nicht der Fall war. Deshalb ist die Abnahme der
Sauerstoffsättigung bei zunehmendem Training ein Indikator des Sauerstoffverbrauchs. Die fortdauernde Einnahme von WS 1442 bewirkt eine Zunahme der Mitochondriendichte in Skelettmuskeln, was zu einer Verschiebung zu oxidativen Typ-1-Fasern führt.

\section{Limitierung}

Die Kontrollgruppe dieser Studie erhielt kein Placebo, weil das Hauptinteresse dem Zusammenhang zwischen den Veränderungen galt, um die zugrunde liegenden Mechanismen und nicht die absoluten Gruppenunterschiede zu erforschen.

Die Studie hat nicht genug Power, um die Wirkung der Behandlung mit WS 1442 zu dokumentieren. Es konnte keine statistische Signifikanz für die beobachteten Behandlungsunterschiede festgestellt werden. Bei allen KCCQItems war die Verbesserung für die Gruppe mit WS 1442 ausgeprägter; für Symptome war die Verbesserung sogar doppelt so gross wie für die Vergleichsgruppe. Dennoch bestand insgesamt kein statistisch signifikanter Unterschied.

\section{Schlussfolgerung}

Patienten mit Herzproblemen und bestehender Auswurffraktion erleben durch ein körperliches Ausdauertraining in Bezug auf Symptome und Lebensqualität einen Nutzen. Die zusätzliche Gabe von WS 1442 zeigt eine Tendenz für eine weitere Verbesserung der Symptome sowie der täglichen Trainingskapazität. Der Extrakt kann problemlos mit Standardbehandlungen von Herzbeschwerden kombiniert werden. 


\section{Literatur}

$>1$ Pittler MH, Guo R, Ernst E: Hawthorn extract for treating heart failure. Cochrane Database Syst Rev 2008;1:CD005312.

-2 Eggeling T, Regitz-Zagrosek V, Zimmermann A, et al: Baseline severity but not gender modulates quantified extract effects in early heart failure - a pooled analysis of clinical trials. Phytomedicine 2011;18:1214-1219.

-3 Peters W, Drüppel V, Kusche-Vihrog K, et al: Nanomechanics and sodium permeability of endothelial surface layer modulated by hawthorn extract WS 1442. PLoS One 2012;7: e29972.

4 Anselm E, Socorro VF, Dal-Ros S, et al: Crataegus special extract WS 1442 causes endothelium-dependent relaxation via a redoxsensitive Src- and Akt-dependent activation of endothelial NO synthase but not via activation of estrogen receptors. J Cardiovasc Pharmacol 2009;53:253-260.

5 Brixius K, Willms S, Napp A, et al: Crataegus special extract WS 1442 induces an endothelium-dependent, NO-mediated vasorelaxation via eNOS-phosphorylation at serine 1177. Cardiovasc Drugs Ther 2006;20:177-184.

-6 Rieckeheer E, Schwinger RH, Bloch W, et al: Hawthorn special extract WS ${ }^{\circledR} 1442$ increases red blood cell NO-fermentation without altering red blood cell deformability. Phytomedicine 2011;19:20-24.
7 Idris-Khodja N, Auger C, Koch E, et al: Crataegus special extract WS ${ }^{\circledR} 1442$ prevents endothelial dysfunction. Phytomedicine 2012;19: 699-706.

8 Schini-Kerth VB: The standardized Crataegus extract WS ${ }^{\circledR} 1442$ prevents systolic hypertension in the DOCA-salt hypertensive rat: benefical effects on target organs (blood vessels, heart and kidney). Personal communication, 2013.

9 Schwinger RH, Pietsch M, Frank K, et al: Crataegus special extract WS 1442 increases force of contraction in human myocardium cAMPindependently. J Cardiovasc Pharmacol 2000; 35:700-707.

10 Hwang HS, Bleske BE, Ghannam MM, et al: Effects of hawthorn on cardiac remodeling and left ventricular dysfunction after 1 month of pressure overloaded-induced cardiac hypertrophy in rats. Cardiovasc Drugs Ther 2008; 22:19-28.

11 Hwang HS, Boluyt MO, Converso K, et al: Effects of hawthorn on the progression of heart failure in a rat model of aortic constriction. Pharmacotherapy 2009;29:639-648.

12 Koch E, Spörl-Aich G: Oral treatment with the Crataegus special extract WS ${ }^{\circledR} 1442$ inhibits cardiac hypertrophy in rats with DOCA-salt or aortic banding induced hypertension. Planta Medica 2006;72:12-15.
13 Chen J, et al: Influence of Crataegus Extract WS $1442^{\circledR}$ on Age-Related Alterations in Skeletal Muscle. Presented at the 78th Annual Meeting of the German Cardiac Society, Mannheim, Germany, 12.4.2012. P696.

14 Gasiorowski A, Dutkiewic ZJ: Comprehensive rehabilitation in chronic heart failure. Am Agric Environ Med 2013;20:606-612.

15 Kitzman DW, Brubaker PH, Herrington DM, et al: Effect of endurance exercise training on endothelial function and arterial stiffness in older patients with heart failure and preserved ejection fraction: a randomized, controlled, single-blind trial. J Am Coll Cardiol 2913;62: 584-592.

16 Nolte K, Herrmann-Lingen K, Wachter R, et al: Effects of exercise training on different quality of life dimensions in heart failure with preserved ejection fraction: the Ex-DHF-P trial. Eur J Prev Cardiol 2015;22:582-593. 